\title{
Correction to: Microglia Interact with Neurons by Forming Somatic Junctions
}

\author{
Yongjie Wang $^{1,2} \cdot$ Zhihui Huang $^{1,2,3}$
}

Published online: 5 June 2020

(C) Shanghai Institutes for Biological Sciences, CAS 2020

\section{Correction to: Neurosci. Bull. https://doi.org/10.1007/s12264-020-00517-3}

The original version of this article unfortunately contained two misunderstandings.

In paragraph 3 of page 3, the sentence starting with "In vivo two-photon imaging revealed that the number of mitochondria significantly increases..." should be "In vivo two-photon imaging revealed that the activity of mitochondria significantly increases...";
The original article can be found online at https:// doi.org/10.1007/s12264-020-00517-3.

Zhihui Huang

hzhzju021@163.com

1 Key Laboratory of Elemene Anti-Cancer Medicine of Zhejiang Province and Holistic Integrative Pharmacy Institutes, Hangzhou Normal University, Hangzhou 311121, China

2 Engineering Laboratory of Development and Application of Traditional Chinese Medicine from Zhejiang Province and Holistic Integrative Pharmacy Institutes, Hangzhou Normal University, Hangzhou 311121, China

3 Department of Neurosurgery, The Affiliated Hospital of Hangzhou Normal University, Hangzhou 310015, China
In paragraph 4 of page 3, the sentence "Microglial process coverage around the somatic junctions was completely abolished after the administration of a P2Y12R inhibitor..." should be "The increased microglial process coverage induced by stroke around the somatic junctions was completely abolished after the administration of a P2Y12R inhibitor ...".

We are sorry for those mistakes. 